\title{
BMJ
}

\section{Retrospective analysis of hospital episode statistics, involuntary admissions under the Mental Health Act 1983, and number of psychiatric beds in England 1996-2006}

\author{
Patrick Keown, consultant psychiatrist and honorary senior lecturer, ${ }^{1}$ Gavin Mercer, consultant \\ psychiatrist, ${ }^{1}$ Jan Scott, professor of psychological medicine ${ }^{2}$
}

${ }^{1}$ East Community Mental Health Team, Molineux Street NHS Centre, Newcastle upon Tyne NE6 1SG

2University Department of Psychiatry, Royal Victoria Infirmary, Newcastle upon Tyne Correspondence to: P Keown p.j.keown@newcastle.ac.uk

Cite this as: BMJ 2008;337:a1837 doi:10.1136/bmj.a1837

\section{ABSTRACT}

Objective To analyse the number of voluntary and involuntary (detentions under the Mental Health Act 1983) admissions for mental disorders between 1996 and 2006 in England.

Design Retrospective analysis.

Setting England.

Main outcome measures Number of voluntary and involuntary admissions for mental disorders in England's health service, number of involuntary admissions to private beds, and number of NHS beds for patients with mental disorders or learning disabilities.

Results Admissions for mental disorders in the NHS in England peaked in 1998 and then started to fall. Reductions in admissions were confined to patients with depression, learning disabilities, or dementia.

Admissions for schizophrenic and manic disorders did not change whereas those for drug and alcohol problems increased. The number of NHS psychiatric beds decreased by $29 \%$. The total number of involuntary admissions per annum increased by $20 \%$, with a threefold increase in the likelihood of admission to a private facility. Patients admitted involuntarily occupied $23 \%$ of NHS psychiatric beds in 1996 but $36 \%$ in 2006.

Conclusions Psychiatric inpatient care changed considerably in the decade from 1996 to 2006, with more involuntary admissions to fewer NHS beds. The case mix has shifted further towards psychotic and substance misuse disorders, which has changed the milieu of inpatient wards. Increasing proportions of involuntary patients were admitted to private facilities.

\section{INTRODUCTION}

Between 1955 and 1995 deinstitutionalisation resulted in the number of beds for mental illness and learning disability in England's health service (NHS) decreasing from over $150000^{1}$ to fewer than 55000 . Although some evidence suggests that crisis teams and services for early intervention in psychosis can reduce the number of admissions for mental disorders compared with traditional psychiatric provision, ${ }^{23}$ one study ${ }^{4}$ found that crisis teams reduce the number of voluntary admissions but not involuntary admissions- detentions under the Mental Health Act 1983. Observers are debating whether a new era of reinstitutionalisation has begun. ${ }^{5}$

We examined changes in the number of psychiatric admissions in England from 1996 to 2006, explored any associations between reductions in NHS bed numbers and involuntary admission rates, and calculated the proportion of involuntary inpatients being treated in non-NHS facilities.

\section{METHODS}

We submitted a request to the NHS Information Centre for data on admissions to NHS hospitals in England from hospital episode statistics. We obtained information on admissions for all mental and behavioural disorders (codes F00-F99, international classification of diseases, 10th revision). To estimate the number of psychiatric beds we combined data on the number of available mental illness and learning disability beds from hospital activity statistics. ${ }^{6}$

Cross sectional data on numbers of involuntary inpatients in NHS hospital and private facilities on 31 March each year were derived from the Department of Health. ${ }^{7}$ We calculated the total number of involuntary admissions per annum by combining the numbers of patients detained under the Mental Health Act - civil, forensic (patients involved in criminal proceedings), and place of safety. Patients whose status changed (from place of safety to civil) were included only once, in the estimates for place of safety. The "count me in census" (the number of patients admitted for mental disorders in NHS and independent hospitals in England and Wales counted on one day each year) provided data on ethnicity of voluntary and involuntary psychiatric inpatients on 31 March 2006. We carried out statistical analyses using Pearson correlations.

\section{RESULTS}

Admissions to NHS hospitals for mental disorders peaked in 1998, at 214326 . Over the study period from 1996 to 2006 , yearly admissions declined by $11 \%$, from 207050 to 183699 . The reductions were confined to 


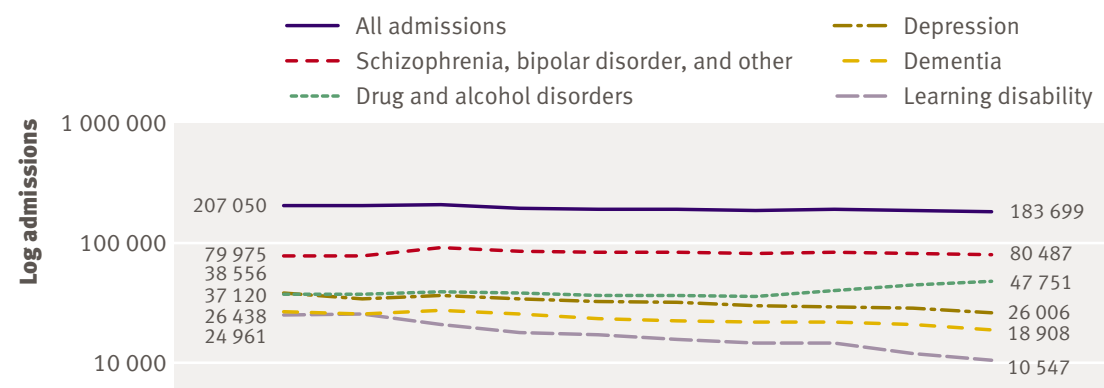

1000



Changes in admission rates (on logarithmic scale) to NHS hospitals for different diagnostic groups of mental disorders, 1996-2006

three subpopulations (figure): patients with learning disabilities (admission decreased by 58\%), unipolar depression $(33 \%)$, or dementia (28\%). Since 2003, admissions for drug and alcohol related disorders increased by $29 \%$, whereas admissions remained relatively stable for other groups-for example, 30639 patients were admitted for schizophreniform disorders in 1996 compared with 30852 in 2006.

Over the decade the total number of involuntary admissions per annum increased by $20 \%$, from 42844 to 51361 ; the number of civil detentions increased by $13 \%$, from 38938 to 43820 , and place of safety detentions increased by $189 \%$, from 2037 to 5877 , whereas forensic detentions decreased by $11 \%$, from 1873 to 1664 . The table shows that on 31 March each year the number of involuntary inpatients in hospital increased by $27 \%$, from 11500 to 14625 , with noticeable increases in the numbers admitted to private facilities compared with NHS facilities (256\% v 12\%). The odds of an involuntary patient being admitted to hospital in a private facility increased threefold (odds ratio $3.2,95 \%$ confidence interval 2.9 to 3.5 ), from 0.06 to $0.21(700 / 10800$ patients $v 2493 / 12132)$.

As the number of psychiatric beds decreased by $29 \%$ (47333 to 33729), the proportion of NHS beds occupied by involuntary patients increased, from $23 \%$ to $36 \%$ (table). A negative correlation was seen between the number of NHS beds and the number of involuntary patients in private facilities $(\mathrm{r}=-0.98$, $\mathrm{P}<0.001)$ or in NHS facilities $(\mathrm{r}=-0.64, \mathrm{P}=0.046)$.

The "count me in census" on 31 March 2006 identified 30023 patients with mental disorders in NHS hospitals and 4262 in private facilities in England; $40 \%$ of NHS inpatients compared with $58 \%$ of private inpatients were involuntary. Ethnic minority groups comprised $21 \%$ of NHS inpatients, $19 \%$ of private inpatients, and $28 \%$ of all involuntary inpatients.

\section{DISCUSSION}

The number of involuntary admissions for mental disorders in England per annum increased by 20\% from 1996 to 2006, whereas the total number of admissions and number of NHS psychiatric beds decreased. Reductions in admissions have largely been confined to patients with depression, learning disability, or dementia. The increase in numbers of involuntary inpatients has been most noticeable in private facilities: in 1996-7 involuntary patients were 15 times more likely to be in an NHS facility than a private facility, but by 2006 only five times more likely.

Limitations of the study are that findings only apply to England and most data sources warned of difficulties in comparing year on year estimates - a problem likely to be amplified by our comparisons across databases. That the data derived from disparate sources indicate similar trends, however, suggests that we are observing real changes in inpatient activity.

These findings support previous studies showing an increase in number of involuntary admissions ${ }^{89}$ against a background of reductions in numbers of NHS beds. Psychotic and affective disorders account for over 50\% of all NHS occupied psychiatric bed days in England; but changes in acute admissions were primarily accounted for by reductions in voluntary admissions for depression, a group with the shortest length of stay (data available on request). Although this may reflect the redirection of patients with depression to crisis teams, the impact on acute inpatient wards has been dramatic, with shifts in case mix (toward psychoses and

\begin{tabular}{|c|c|c|c|c|c|c|c|c|c|c|c|c|}
\hline \multicolumn{13}{|c|}{ Number of beds for mental illness and learning disability, and number of involuntary inpatients between 1996 and 2006, in England } \\
\hline Variable & $1996-7$ & $1997-8$ & $1998-9$ & $\begin{array}{l}1999- \\
2000\end{array}$ & 2000-1 & $2001-2$ & $2002-3$ & $2003-4$ & 2004-5 & $2005-6$ & $\%$ change & $\begin{array}{l}\text { Pearson } \\
\text { correlation* }\end{array}$ \\
\hline $\begin{array}{l}\text { No of NHS beds for mental } \\
\text { disorders and learning } \\
\text { disability }\end{array}$ & 47333 & 44798 & 43183 & 41007 & 40530 & 38477 & 37791 & 37464 & 35701 & 33729 & $29 \%$ reduction & - \\
\hline \multicolumn{13}{|l|}{$\begin{array}{l}\text { Involuntary inpatients on } 31 \\
\text { March: }\end{array}$} \\
\hline No in NHS facilities & 10800 & 11800 & 11823 & 11473 & 12150 & 11566 & 11589 & 11708 & 12148 & 12132 & $12 \%$ increase & $r=-0.64 ; P=0.046$ \\
\hline No in private facilities & 700 & 850 & 1170 & 1382 & 1679 & 1893 & 1999 & 2292 & 2533 & 2493 & $256 \%$ increase & $r=-0.98 ; P<0.001$ \\
\hline Total No & 11500 & 12700 & 12993 & 12855 & 13829 & 13459 & 13588 & 14000 & 14681 & 14625 & $27 \%$ increase & $r=-0.94 ; P<0.001$ \\
\hline $\begin{array}{l}\% \text { of NHS beds occupied by } \\
\text { involuntary inpatients } \dagger\end{array}$ & 23 & 26 & 27 & 28 & 30 & 30 & 31 & 31 & 34 & 36 & - & - \\
\hline
\end{tabular}




\section{WHAT IS ALREADY KNOWN ON THIS TOPIC}

Rates of involuntary admissions under the Mental Health Act 1983 increased in the 1980s and '90s

The number of psychiatric beds in the NHS has been decreasing since the $1950 \mathrm{~s}$

\section{WHAT THIS STUDY ADDS}

The number of patients admitted to the NHS with mental disorders peaked in 1998 and is now decreasing

The number of psychiatric beds in the NHS has continued to fall and rates of involuntary admissions have continued to rise

A greater proportion of psychiatric inpatients are now involuntary and are being treated in private facilities and substance misuse disorders, which has changed the milieu on inpatient psychiatric wards.

Reuse of information from the "count me in census" and other datasets was covered by the public sector information licence held by Newcastle University.

Contributors: All authors were involved in the conception of the study and interpretation of the data. PK collected the data and wrote the first draft, and is the guarantor. JS revised drafts of the paper. All authors were involved in approving the final draft to be published.

Funding: None

Competing interests: JS has received funding for continuing medical education talks on psychosocial aspects of bipolar disorders, unrestricted educational grants for research on medication adherence, and been a member of advisory boards for Astra Zeneca, BMS Otsuka, Eli Lilly, GSK, Jansen Cilag, and Sanofi-Aventis. PK has received funding for continuing medical education talks from Jansen Cilag. PK and GM are both employed by the NHS. JS's clinical practice is entirely within the NHS

Ethical approval: Not required

Provenance and peer review: Not commissioned; externally peer reviewed.

(involuntary inpatients).

The increase in number of involuntary admissions to private facilities is noteworthy as the NHS purchases about $80 \%$ of private psychiatric provision. One of the largest growth areas has been in the provision of private medium secure beds even though NHS forensic facilities have expanded. The decrease in forensic involuntary admissions is therefore surprising. A question for further research is whether the changes identified in this study have applied across demographic and diagnostic groups and NHS regions for the duration of the Mental Health Act 1983.

\section{Conclusions}

Psychiatric inpatient care changed considerably from 1996 to 2006 , with more involuntary patients admitted to fewer NHS beds and increasing proportions of involuntary patients admitted to private facilities. The decrease in acute general adult admissions has been confined to voluntary patients with depression. The inpatient case mix has shifted further towards psychotic
1 Davidge M, Elias S, Jayes B, Wood K, Yates J. Survey of English mental illness hospitals. Birmingham: Health Service Management Centre, 1993.

2 Glover G, Arts G, Babu KS. Crisis resolution/home treatment teams and psychiatric admission rates in England. Br J Psychiatry 2006;189:441-5.

3 Craig TKJ Garety P, Power P, Rahaman N, Colbert S, Fornells AM, et al. The Lambeth Early Onset (LEO) Team: randomised controlled trial of the effectiveness of specialised care for early psychosis. BMJ 2004;329:1067-71.

4 Johnson S, Nolan F, Pilling S, Sandor A, Hoult J, McKenzie N, et al. Randomised controlled trial of acute mental health care by a crisis resolution team: the north Islington crisis study. BMJ 2005;331:4.

5 Priebe S, Turner T. Reinstitutionalisation in mental health care. $B M$ 2003;326:175-6.

6 Department of Health. Bed availability and occupancy, England. London: DoH, 2006.

7 Information Centre. Inpatients formally detained in hospitals under the Mental Health Act 1983 and other legislation. London: Government Statistical Service, 2007

8 Priebe S, Badesconyi A, Fioritti A, Hansson L, Kilian R, comparison of data on service provision from six European countries. BMI 2005;330:123-6.

9 Wall S, Hotopf M, Wessely S, Churchill R. Trends in the use of the Mental Health Act: England, 1984-96. BMJ 1999;318:1520-1.

Accepted: 2 August 2008 Torres-Gonzales F, et al. Reinstitutionalisation in mental health care: 